\title{
FINITE-DIMENSIONAL DIFFERENTIAL ALGEBRAIC GROUPS AND THE PICARD-VESSIOT THEORY
}

\author{
ANAND PILLAY \\ Department of Mathematics, University of Illinois at Urbana-Champaign \\ Altgeld Hall, 1409 W. Green St., Urbana, IL 61801, U.S.A. \\ E-mail: pillay@math.uiuc.edu \\ and \\ Institut für Mathematik, Humboldt Universität, D-10099 Berlin, Germany
}

\begin{abstract}
We make some observations relating the theory of finite-dimensional differential algebraic groups (the $\partial_{0}$-groups of [2]) to the Galois theory of linear differential equations. Given a differential field $(K, \partial)$, we exhibit a surjective functor from (absolutely) split (in the sense of Buium) $\partial_{0}$-groups $G$ over $K$ to Picard-Vessiot extensions $L$ of $K$, such that $G$ is $K$-split iff $L=K$. In fact we give a generalization to " $K$-good" $\partial_{0}$-groups. We also point out that the "Katz group" (a certain linear algebraic group over $K$ ) associated to the linear differential equation $\partial Y=A Y$ over $K$, when equipped with its natural connection $\partial-[A,-]$, is $K$-split just if it is commutative.
\end{abstract}

1. Introduction. Let $(K, \partial)$ be a differential field of characteristic 0 with algebraically closed field $k$ of constants. Let

$$
\partial Y=A Y
$$

be a linear differential equation over $K$. That is, $Y$ is an $n$ by 1 column vector of indeterminates and $A$ is a $n$ by $n$ matrix over $K$. Let $(L, \partial)$ be a Picard-Vessiot extension for (*). The differential Galois group $\operatorname{Aut}_{\partial}(L / K)$ is well-known to have the structure of an algebraic subgroup of $G L(n, k)$, so the group of $k$-points of some linear algebraic group $G_{k}$ over $k$. On the other hand, another group $G_{K}^{\prime}$, a linear algebraic subgroup now over $K$, was defined in [5] via the Tannakian point of view. The current paper was in part motivated by an informal question of Daniel Bertrand regarding the differential algebraic meaning of $G_{K}^{\prime}$. In fact the Tannakian theory already equips $G_{K}^{\prime}$ with a "connection" $\nabla: \partial-[A,-]$, giving it the structure of a $\partial_{0}$-group over $K$ (see section 2 for the definitions). The group of $L$-points (or even $\hat{K}$-points for $\hat{K}$ a differential closure of $K$ ) of this

2000 Mathematics Subject Classification: Primary 12H05; Secondary 34M60.

Partially supported by an NSF grant and a Humboldt Foundation Research Award.

The paper is in final form and no version of it will be published elsewhere. 
$\partial_{0}$-group $\left(G_{K}^{\prime}, \nabla\right)$ (see Definition 2.4) acts on the solution space of $(*)$ in $L$ and should be viewed as the real "intrinsic" differential Galois group of $(*) .\left(G_{K}^{\prime}, \nabla\right)$ is isomorphic over $L$ to $G_{k}$ equipped with the trivial connection, so is (absolutely) split in the sense of Buium [2]. We will point out that $\left(G_{K}^{\prime}, \nabla\right)$ is $K$-split (that is, isomorphic over $K$ to an algebraic group over $k$ equipped with the trivial connection) just if $G_{K}^{\prime}$ is commutative. In particular $\left(\left(G_{K}^{\prime}\right)^{0}, \nabla\right)$ is $K^{a l g}$-split iff $\left(G_{K}^{\prime}\right)^{0}$ is commutative.

On the other hand we will point out that any $\partial_{0}$-group $(G, \nabla)$ which is defined over $K$ and absolutely split, gives rise in a natural way to a Picard-Vessiot extension $L$ of $K$ : essentially $L$ will be generated over $K$ by a canonical parameter for an isomorphism of $(G, \nabla)$ with an algebraic group over $k$ equipped with the trivial connection. Moreover any Picard-Vessiot extension of $K$ arises in this way: given the equation $(*)$ above, a fundamental matrix $U$ of solutions of $(*)$ will be a canonical parameter for an isomorphism between $\left(G_{a}^{n}, \partial-A\right)$ and $G_{a}^{n}$ equipped with the trivial connection.

The observations in this paper are not too difficult. In fact the paper should be seen as an introduction to the Kolchin-Cassidy-Buium (and model-theoretic) theory of $\partial_{0}$-groups, for those familiar with the Picard-Vessiot theory (Galois theory of linear differential equations). Concerning the general theory of $\partial_{0}$-groups our only innovation is to bring into the picture rationality issues, the notion of being split over $K$, where $K$ is an arbitrary (not necessarily differentially closed) differential field.

The rest of the paper is devoted to filling in the details of the above observations. At some point in section 3 some model-theoretic notation is used. The reader is referred to [11] for explanations.

I would like to thank Daniel Bertrand for his original questions, his interest in the answers, as well as for his beautifully concise and informative review [1] of Magid's excellent book "Lectures on differential Galois theory". Thanks also to Wai-Yan Pong for several helpful discussions.

2. $\partial_{0}$-groups. The differential algebraic groups (of Kolchin [7]) are essentially just the group objects in Kolchin's category of differential algebraic varieties. From the modeltheoretic point of view they are definable groups in a differentially closed field (see [11]). Such a group is said to be "finite-dimensional" if the differential function field of its connected component has finite transcendence degree (over the universal domain say). Finite-dimensional differential algebraic groups were exhaustively studied by Buium [2].

It will be convenient to introduce finite-dimensional differential algebraic groups $\left(\partial_{0^{-}}\right.$ groups) via a different formalism, that of Buium's "algebraic $D$-groups": a $\partial_{0}$-group will be an algebraic group equipped with what I will loosely refer to as a "connection". Let $(K, \partial)$ be a differential field (of characteristic 0 ), with field $C_{K}=k$ of constants.

Definition 2.1. A $\partial_{0}$-group over $K$ is a pair $(G, \nabla)$ where $G$ is an algebraic group over $K$ and $\nabla$ is an extension of $\partial$ to a derivation of the structure sheaf $O_{K}(G)$ of $G$, commuting with co-multiplication. A homomorphism (over $K$ ) between $\left(G_{1}, \nabla_{1}\right)$ and $\left(G_{2}, \nabla_{2}\right)$ is the obvious thing (a $K$-homomorphism $f$ of algebraic groups such that the corresponding map $f^{*}$ between structure sheaves respects the respective derivations). 
Rather quickly we will replace the derivation $\nabla$ by a more accessible object. Given an algebraic group $G$ over $K, T(G)$ will denote the tangent bundle of $G$, another algebraic group over $K . \tau(G)$ is a twisted version of $T(G)$ taking into account the derivation $\partial$ of $K$ : working locally, if $G$ is defined by polynomial equations $P_{j}\left(X_{1}, \ldots, X_{n}\right)=0$, then $\tau(G)$ is defined by the equations $\sum_{i=1}^{n} \partial P_{j} / \partial X_{i}\left(X_{1}, \ldots, X_{n}\right) Y_{i}+P_{j}^{\partial}\left(X_{1}, \ldots, X_{n}\right)=0$, where $P^{\partial}$ is the result of applying $\partial$ to the coefficients of $P . \tau(G)$ has naturally the structure of an algebraic group over $K$ with a surjective homomomorphism $\pi$ to $G$ (see [8]). If $G$ is defined over $k$ (the constants of $K$ ) then $\tau(G)$ identifies with $T(G)$ and $\pi$ is as usual. A $K$-rational homomorphism $f$ from $G_{1}$ to $G_{2}$ yields a $K$-rational homomorphism $\tau(f): \tau\left(G_{1}\right) \rightarrow \tau\left(G_{2}\right)$ commuting with $\pi$.

REMARK 2.2. If $G$ is an algebraic group over $K$, then a $\partial_{0}$-group structure on $G$ (that is, a derivation $\nabla$ as in Definition 2.1) is equivalent to a $K$-rational homomorphic section $s: G \rightarrow \tau(G)$ of $\pi: \tau(G) \rightarrow G$.

Proof. This is immediate: given a point $a$ of $G, \nabla$ determines a derivation of the local ring at $a$, yielding a point $s(a)$ in the fibre $\tau(G)_{a} . \nabla$ commuting with co-multiplication is equivalent to $s$ being a homomorphism.

Thus a $\partial_{0}$-group over $K$ can be identified with a pair $(G, s)$ where $G$ is an algebraic group over $K$ and the "connection" $s: G \rightarrow \tau(G)$ is a $K$-rational homomorphic section of $\pi$. A $K$-homomorphism $f$ between such $\left(G_{1}, s_{1}\right)$ and $\left(G_{2}, s_{2}\right)$ is then a $K$-rational homomorphism $f$ of algebraic groups such that $\tau(f) . s_{1}=s_{2} . f$. If $G$ happens to be defined over the constants $k$ of $K$ then as mentioned above $\tau(G)=T(G)$, and we have at our disposal the "trivial connection" $s_{0}$, namely $s_{0}$ is the 0 -section of $T(G)$.

Definition 2.3. Let $(G, s)$ be a $\partial_{0}$-group over $K$. We say that $(G, s)$ is $K$-split if it is isomorphic over $K$ to some $\left(G_{0}, s_{0}\right)$ where $G_{0}$ is defined over $k$ and $s_{0}$ is the trivial connection.

Note that if $X$ is a variety defined over $K$ and $a \in X(\mathcal{U})$ is a $\mathcal{U}$-rational point of $X$ then the expression $\partial(a)$ makes sense: working in an affine neighbourhood of $a$, defined over $K$, just apply $\partial$ to the coordinates of $a$. Moreover $\partial(a)$ is in $\tau(X)_{a}$.

Definition 2.4. Let $(G, s)$ be a $\partial_{0}$-group over $K$. Let $(L, \partial)$ be a differential field extension of $K$. The group $(G, s)(L)$ of $L$-rational points of $(G, s)$ is $\{g \in G(L): \partial(g)=s(g)\}$.

Let $(\mathcal{U}, \partial)$ be a universal domain, that is, a differentially closed field containing $K$, of cardinality $\kappa>$ the cardinality of $K$, with the following properties: (i) any isomorphism between small (of cardinality $<\kappa$ ) differential subfields of $\mathcal{U}$ extends to a (differential) automorphism of $\mathcal{U}$, and (ii) if $K_{1}<K_{2}$ are small differential fields then any embedding of $K_{1}$ in $\mathcal{U}$ extends to an embedding of $K_{2}$ in $\mathcal{U}$.

If $(G, s)$ is a $\partial_{0}$-group over $K$, then $(G, s)(\mathcal{U})$, the set of points of $(G, s)$ in $\mathcal{U}$, is a finite-dimensional differential algebraic group, defined over $K$, in the sense of Kolchin. Moreover any finite-dimensional differential algebraic group arises in this way (see [2]). We will often identify $(G, s)$ with its group of $\mathcal{U}$ points, or sometimes with its group of $\hat{K}$-points where $\hat{K}$ is a differential closure of $K$. Also any $\partial_{0}$-group over $K$ can naturally be also considered as a $\partial_{0}$-group over $\mathcal{U}$ (or over any differential field extending $K$ ). 
Definition 2.5. The $\partial_{0}$-group $(G, s)$ over $K$ is said to be (absolutely) split if it is $\mathcal{U}$-split, equivalently if it is $L$-split for some differential field $L>K$.

Note that $(G, s)$ (over $K$ ) is absolutely split iff it is $\hat{K}$-split. In [2], Buium begins (and almost completes) the classification of (connected) $\partial_{0}$-groups over $\mathcal{U}$ : the issue being to first determine which (connected) algebraic groups $G$ over $\mathcal{U}$ have some " $D$-group" structure, that is, can be equipped with a suitable $s$, and secondly, to note that the space of $D$-group structures on $G$ is, if nonempty, a principal homogeneous space for the set of rational homomorphic sections of the tangent bundle of $G$. It would be of interest to try to classify the $\partial_{0}$-groups over a given (say algebraically closed) differential field $K$, up to $K$-isomorphism, although possibly this is already implicit in Buium's work. In any case, one of the points of the current paper is that split but non- $K$-split $\partial_{0}$-groups over $K$ are closely bound up with Picard-Vessiot extensions of $K$. This will be discussed in the next section. For the rest of this section I will give some examples and elementary facts about $D$-group structures on commutative algebraic groups over the constants, working over $\mathcal{U}$. $C$ denotes the field of constants of $\mathcal{U}$. Note first that for such $G$ any section $s: G \rightarrow T(G)$ can be identified with a homomorphism from $G$ into its Lie algebra. (Canonically $T(G)=L(G) \times G$, so $s=(f, i d)$ for a unique $f: G \rightarrow L(G)$.)

EXAmple 2.6 ( $D$-group structures on commutative unipotent groups). Let $G=G_{a}^{n}$. A rational homomorphism from $G$ to $L(G)$ is precisely a linear map from $G$ to itself. Thus each $D$-group structure on $G$ has the form $\left(G, s_{A}\right)$ for some $n$ by $n$ matrix $A$ over $\mathcal{U}$, where $s_{A}$ is left matrix multiplication by $A$. Each such $\partial_{0}$-group is split (over $\mathcal{U}$ ): The set of $\mathcal{U}$-points of $\left(G, s_{A}\right)$ is an $n$-dimensional vector space over $C$. Let $b_{1}, \ldots, b_{n}$ (thought of as column vectors) be a $C$-basis. Matrix multiplication by $\left(b_{1}, \ldots, b_{n}\right)$ yields an isomorphism between $\left(G, s_{0}\right)$ and $\left(G, s_{A}\right)$. This isomorphism need not be defined over the differential field generated by the coordinates of $A$.

EXAMPLE 2.7 ( $D$-group structures on semiabelian varieties over the constants). Let $A$ be a semiabelian variety over $C$. As above, $D$-group structures $s$ on $A$ are given by rational homomorphisms from $A$ to the Lie algebra of $A$, of which there is only one, the 0 map. So the 0 -section is the unique $D$-group structure on $A$.

EXAMPLE 2.8 ( $D$-group structures on commutative algebraic groups over the constants). Let $G$ be a connected commutative algebraic group defined over $C$. We will prove a special and easy case of a result from [2]: Let $(G, s)$ be a $D$-group structure (over $\mathcal{U}$ ) on $G$. Then $(G, s)$ is split if and only if the unipotent radical $U$ of $G$ (which note is also defined over $C$ ) is a $D$-subgroup of $G$ (that is, $s(U)$ is contained in $L(U)$ ). Right to left is clear. Suppose now that $s$ takes $U$ into $L(U)$.

Claim. $s(G)=s(U)$.

Proof. Otherwise $s$ induces a nonconstant homomorphism from the semiabelian variety $G / U$ into $L(G) / L(U)$ which is impossible.

Let $H<G$ be the kernel of $s$. Using Example 2.6 we can write $U$ as a direct sum of $H \cap U$ and a $D$-subgroup $U_{1}$ of $U$. By the claim $G$ is the direct sum of $H$ and $U_{1}$. As $U_{1}$ is split (by Example 2.6), $G$ is split. 
ExAmple 2.9 (A nonsplit $D$-group structure on $G_{m} \times G_{a}$.). Let $G=G_{m} \times G_{a}$. $T(G)=\tau(G)$ consists of the set of $(x, y, u, v)$ where $x \neq 0$, and has group structure given by: $\left(x_{1}, y_{1}, u_{1}, v_{1}\right) \cdot\left(x_{2}, y_{2}, u_{2}, v_{2}\right)=\left(x_{1} x_{2}, y_{1}+y_{2}, u_{1} x_{2}+u_{2} x_{1}, v_{1}+v_{2}\right)$. $\pi: T(G) \rightarrow G$ is projection on the first two coordinates. Let $s: G \rightarrow T(G)$ be: $s(x, y)=(x, y, x y, 0)$. Then $s$ is a section of $\pi$ as well as being a homomorphism. $(G, s)(\mathcal{U})$ $=\{(x, y) \in G: \partial x=x y, \partial y=0\}$, which is isomorphic to the differential algebraic subgroup $\left\{x \in G_{m}: \partial(\partial x / x)=0\right\}$ of $G_{m}$.

Rather deeper results concern $D$-group structures on algebraic groups which cannot be defined over the constants. For example, an abelian variety $A$ over $\mathcal{U}$ which is not isomorphic to an abelian variety over $C$ has no $D$-group structure. $A$ will nevertheless have finite-dimensional differential algebraic subgroups (defined by differential equations of order $>1$ ) which correspond to $D$-group structures on extensions of $A$ by unipotent groups. (See [2] and [8].) Such examples will not concern us too much in this paper. Moreover, the further away from the constants an algebraic group $G$ is, the more rigid will be the space of $D$-group structures on $G$.

3. Relations with the Picard-Vessiot theory. We will take as our basic reference Bertrand's review [1]. Recall the basic set-up: $K$ is a differential field (considered as a small subfield of $\mathcal{U}$ ) with algebraically closed field $k$ of constants.

$$
\partial Y=A Y
$$

is a linear ODE over $K . V^{\partial}$ denotes the solution space of $(*)$ in $\mathcal{U}$, an $n$-dimensional vector space over $C$ (the constants of $\mathcal{U}) . V^{\partial}(\hat{K})$ denotes the vectors in $V^{\partial}$ whose coordinates are in $\hat{K} \cdot V^{\partial}(\hat{K})$ is an $n$-dimensional vector space over $k$. The Picard-Vessiot extension $L / K$ for $(*)$ is the (differential) field generated over $K$ by the coordinates of elements of $V^{\partial}(\hat{K})$. (As $\hat{K}$ has the same constants as $K, L$ has the same constants as $K$.) Let us fix a fundamental solution matrix $U$ for $(*)$, namely the columns of $U$ form a basis for $V^{\partial}(\hat{K})$ over $k$ (and so also for $V^{\partial}$ over $C$ ). Via $U$ we obtain an isomorphism $\rho_{U}$ between $A_{u t}(L / K)$ and an (algebraic) subgroup of $G L(n, k): \sigma(U)=U \rho_{U}(\sigma)$. Write this subgroup as $G_{k}(k)$, the group of $k$-rational points of a linear algebraic group $G_{k}$ over $k$. Note that $G_{k}(k)$ is precisely the set of $\hat{K}$-points of the $\partial_{0}$-group $\left(G_{k}, s_{0}\right)$ where $s_{0}$ is the trivial connection.

In [5], a somewhat different definition of the Galois group of $(*)$ was given, but now as an algebraic group $G_{K}^{\prime}$ over $K$. This was defined via the Tannakian theory. The usual notion of a connection on a vector space over the differential field $(K, \partial)$ is an additive endomorphism $D: V \rightarrow V$ such that for any $\lambda \in K$, and $v \in V, D(\lambda v)=\partial(\lambda) v+\lambda D(v)$. Let $V=K^{n}$. From the equation $(*)$ we obtain a connection $D_{V}: \partial-A$ on $V . D_{V}$ induces, on each $K$-vector space $E$ constructed from $V$ by iterating direct sums, tensor products, and duals, a connection $D_{E} \cdot G L(n, K)$ acts on each of these vector spaces, and $G_{K}^{\prime}$ is defined to be $\{g \in G L(n, K): g(W)=W$, for every $K$-subpace $W$ of any construction $E$ over $V$ for which $\left.D_{E}(W) \subseteq W\right\}$.

Note that $G L(n, K)$ acts on itself and thus on its own coordinate ring $R$ over $K$. As remarked in [1], $G_{K}^{\prime}$ is precisely the stabilizer of the ideal $I \subset R$ consisting of polynomials 
which vanish on the fundamental matrix $U$ of solutions of $(*)$. (And this does not depend on the choice of $U$.) In any case we obtain an algebraic group over $K$, and one can ask in what sense $G_{K}^{\prime}$ is the Galois group of $(*)$. Note that $G_{K}^{\prime} \subseteq \operatorname{End}(V)=V \otimes V^{*}$ and the latter $K$-vector space, itself a construction over $V$, is equipped with the connection $D_{\operatorname{End}(V)}: \partial-[A,-]$ (that is, for $X \in \operatorname{End}(V), D_{\operatorname{End}(V)}(X)=\partial X-[A, X]$ ). (This connection is also implicit in [3]). In any case this connection equips $G_{K}^{\prime}$ with the structure of a $\partial_{0}$-group $\left(G_{K}^{\prime}, s\right)$ where $s(g)=[A, g]$. It is this $\partial_{0}$-group (or rather its group of $\hat{K}$ points), which should be considered as the canonical (or intrinsic) Galois group of $(*)$. At this point we make use of model-theoretic/differential algebraic language. Working in $\mathcal{U}, t p(-/ K)$ means type over $K$ in the sense of differential fields, and $t p_{f}(-/ K)$ means type over $K$ in the sense of fields. Let $G_{1}=\left(G_{K}^{\prime}, s\right)(\mathcal{U})$.

REMARK 3.1. $G_{1}=\left\{g \in G L(n, \mathcal{U}): \partial g=[A, g]\right.$ and for any $U_{1} \in G L(n, \mathcal{U})$ realising $\operatorname{tp}_{f}(U / K)$ and independent (in the sense of fields) from $g$ over $K, \operatorname{tp}_{f}\left(g U_{1} / K\right)=$ $\left.t p_{f}\left(U_{1} / K\right)\right\}$.

\section{Proof. Clear.}

Lemma 3.2. $G_{1}$ acts faithfully (by left matrix multiplication) on $V^{\partial}$. Moreover this action is precisely the group of permutations of $V^{\partial}$ induced by automorphisms of the differential field $\mathcal{U}$ which fix $K \cup C$ pointwise.

Proof. We start with

Claim. Let $g \in G L(n, \mathcal{U})$. Then $g \in G_{1}$ if and only if $\operatorname{tp}(g U / K \cup C)=t p(U / K \cup C)$.

Proof. Note first that $t p(U / K)=r(x)$ say is determined by (i) $t p_{f}(U / K)=r_{f}(x)$, and (ii) $\partial x=A x$. Note also that $r(x)$ has a unique extension to a complete type $r^{\prime}(x)$ say, over $K \cup C$ (otherwise there would be new constants in $L=K(U)$, which there are not).

Suppose first that $t p(g U / K)=r$. Let $U_{1}$ realise $r$ independently from $g$ over $K$ in the sense of differential fields. As $U$ and $U_{1}$ are bases for $V^{\partial}$ over $C, U_{1}=U B$ for some $B \in G L(n, C)$. Now $U, g U$ and $U_{1}$ each realise $r^{\prime}$ (over $K \cup C$ ). It follows that $g U_{1}=g(U B)=(g U)(B)$ also realizes $r^{\prime}$, in particular $r$. As $U_{1}$ is independent from $g$ over $K$ in the sense of fields, and (as $\partial\left(U_{1}\right)=A U_{1}$ and $\left.\partial\left(g U_{1}\right)=A\left(g U_{1}\right)\right) \partial(g)=[A, g]$, we see from Remark 3.1 that $g \in G_{1}$.

The other direction of the Claim follows by reversing the argument.

The claim gives us a bijection between the set of permutations $\sigma$ of $V^{\partial}$ induced by $A u t_{\partial}(\mathcal{U} / K \cup C)$ and $G_{1}: \sigma$ goes to $g$ where $\sigma(U)=g U$. In fact the action of $\sigma$ on $V^{\partial}$ is identical to the action of $g$ by left matrix multiplication: if $v \in V^{\partial}$ (a column vector), then $v=U c$ for some column vector of constants, and $\sigma(v)=\sigma(U c)=\sigma(U) c=(g U) c=$ $g(U c)=g v)$. The map $(\sigma$ to $g)$ is clearly a group isomorphism.

Corollary 3.3. $G_{1}(\hat{K})\left(=\left(G_{K}^{\prime}, s\right)(\hat{K})\right)$ acts on $V^{\partial}(\hat{K})$ (by left matrix multiplication) inducing an isomorphism with $\operatorname{Aut}_{\partial}(L / K)$.

Proof. The first part is immediate from the lemma, using the fact that $\hat{K}$ is homogeneous over $K$ in the model-theoretic sense. As $L$ is generated over $K$ by the points of $V^{\partial}(\hat{K})$ the second part also follows. 
REMARK 3.4. $G_{1}$ as above is also the intrinsic definable automorphism group of $V^{\partial}$ over $C$ in the model-theoretic sense.

Explanation. If $P$ and $Q$ are $\emptyset$-definable sets in a saturated model $M$ of a stable theory and $P$ is $Q$-internal, then the group $(G, P)$ of permutations of $P$ induced by automorphisms of $M$ which fix $Q$ pointwise, is isomorphic to some definable (in $M$ ) group action on $P$. This is due in full generality to Hrushovski [4], and an exposition appears in chapter 7 of [10]. The Picard-Vessiot theory is a special case, as (working over $K$ ), $V^{\partial}$ is $C$-internal. (In fact Poizat [12] was the first to give a model-theoretic explication of the Picard-Vessiot theory and Kolchin's more general strongly normal theory.) However, even in the general model-theoretic context, there are various incarnations of the definable automorphism group and its action on $P$ : the intrinsic case is where $G$ and its action are $\emptyset$-definable and $G$ lives in $P^{e q}$. The other case depends on the choice of a "fundamental set of solutions" $u$ from $P: G$ lives in $Q^{e q}$, is defined over the canonical base of $t p(u / Q)$ and in general requires the parameter $u$ to define its action on $P$. In any case, transplanted to the Picard-Vessiot situation, it is $\left(G_{K^{\prime}}, s\right)$ which is the intrinsic group, and $\left(G_{k}, s_{0}\right)$ ( $s_{0}$ being the trivial connection) which is the non-canonical group.

Note that the $\partial_{0}$-group $\left(G_{K}^{\prime}, s\right)$ (where $\left.s(-)=[A,-]\right)$ is $L$-split. It is isomorphic to $\left(G_{k}, s_{0}\right)$ by the map $\rho_{U}: g U=U \rho_{U}(g)$. Note also that we obtain easily a simple definition of $G_{k}$ as an algebraic group over $k$ : Let $U_{1}, \ldots, U_{s}$ realize the distinct nonforking extensions of $t p(U / K)$ over $K(U)$. Let $U_{i}=U B_{i}$ for $B_{i} \in G L(n, C)$. Let $p_{i}=t p_{f}\left(B_{i} / k\right)$. Then $G_{k}$ is precisely the stabilizer of $\left\{p_{1}, \ldots, p_{s}\right\}$ in $G L(n)$.

Note that the set $X$ of realizations of $t p(U / K)$ is a left principal homogeneous space for $G_{1}\left(=\left(G_{K}^{\prime}, s\right)(\mathcal{U})\right)$, and a right principal homogeneous space for $G_{2}=G_{k}(C)(=$ $\left(G_{k}, s_{0}\right)(\mathcal{U})$ ) (and likewise working with $\hat{K}$-rational points), where the actions commute $\left(g(x h)=(g x) h\right.$ for $\left.g \in G_{1}, x \in X, h \in G_{2}\right)$. That is, $X$ is a (differential algebraic) bi-torsor for $\left(G_{1}, G_{2}\right)$ defined over $K$. It follows that $G_{1}$ is isomorphic over $K$ to $G_{2}$ just if $G_{1}$ (so also $G_{2}$ ) is commutative. This kind of thing (in the general model-theoretic framework of definable automorphism groups) was already observed in passing in [4]. In any case we will give some details.

Let us start with a general lemma:

Lemma 3.5. Let $\left(H_{1}, X, H_{2}\right)$ be an abstract bi-torsor. That is, $X$ is an (abstract) left principal homogeneous space for the (abstract) group $H_{1}$, an (abstract) right principal homogeneous space for the (abstract) group $\mathrm{H}_{2}$ and the left and right actions commute. For $x \in X$, let $\rho_{x}$ be the isomorphism between $H_{1}$ and $H_{2}$ defined by $h x=x \rho_{x}(h)$. Let $h \in H_{1}$. The following are equivalent:

(i) $h$ is in the centre of $H_{1}$.

(ii) for all $x \in X, \rho_{x}=\rho_{h x}$.

(iii) for some $x \in X, \rho_{x}=\rho_{h x}$.

Proof. (i) implies (ii). Assume $h \in Z\left(H_{1}\right)$. Let $g \in H_{1}$ and $x \in X$. Then $g h x=$ $x \rho_{x}(g) \rho_{x}(h)$ and $h g x=x \rho_{x}(h) \rho(g)$. But also $g h x=(h x) \rho_{h x}(g)=x \rho_{x}(h) \rho_{h x}(g)$. So as $g h=h g$ we see that $\rho_{h x}(g)=\rho_{x}(g)$. As $g \in H_{1}$ was arbitrary, we see that $\rho_{h x}=\rho_{x}$.

(ii) implies (iii) is immediate. 
(iii) implies (i) follows by reversing the proof of (i) implies (ii).

Let us now return to the differential situation: $L$ is the Picard-Vessiot extension of $K$ for the equation $\partial Y=A Y$ over $K, G_{K}^{\prime}$ is the Katz group and $s(-)$ is $[A,-] . U$ is a fundamental matrix of solutions of $(*)$ (and $L=K(U)$ ).

Corollary 3.6. $\left(G_{K}^{\prime}, s\right)$ is $K$-split if and only if $G_{K}^{\prime}$ is commutative.

Proof. Recall the notation: $G_{1}=\left(G_{K}^{\prime}, s\right)(\mathcal{U}), G_{2}=G_{k}(C)$, and let $X$ be the space of realisations of $t p(U) . \rho_{U}$ is the isomorphism between $G_{1}$ and $G_{2}: g U=U \rho_{U}(g)$.

Firstly, let us suppose that $G_{K}^{\prime}$ is commutative. Then so is $G_{1}$ and by the previous lemma, $\rho_{U}=\rho_{g U}$ for all $g \in G_{1}$. But $X$ is precisely the set of such $g U\left(g \in G_{1}\right)$, so $\rho_{U}$ is fixed by $K$-automorphisms of the differential field $\mathcal{U}$ so is defined over $K:\left(G_{K}^{\prime}, s\right)$ is $K$-split.

Conversely, suppose $\left(G_{K}^{\prime}, s\right)$ is $K$-split. So there is a $K$-definable isomorphism $f$ between $G_{1}$ and some $\partial_{0}$-group $G_{3}$ of the form $\left(H, s_{0}\right)$ where $H$ is an algebraic group over $C$ and $s_{0}$ is the trivial connection. Then $H$ must be defined over $k . \rho_{U} \cdot f^{-1}$ is then an isomorphism between $G_{3}$ and $G_{2}$ defined over $\hat{K}$. As both $G_{3}$ and $G_{2}$ are the groups of $C$-points of algebraic groups defined over the algebraically closed field $k$, and $k$ is the constants of $\hat{K}$, it follows that $\rho_{U} \cdot f^{-1}$ is defined over $k$ (and is actually an isomorphism of algebraic groups). Thus (the differential algebraic isomorphism) $\rho_{U}$ is defined over $K$. So for each $g \in G_{1}, \rho_{U}=\rho_{g U}$. By Lemma 3.5, $G_{1}$ is commutative. But easily $G_{1}$ is Zariski-dense in $G_{K}^{\prime}$, whereby $G_{K}^{\prime}$ is commutative.

With the same notation:

Corollary 3.7. $\left(\left(G_{K}^{\prime}\right)^{0}, s\right)$ is $K^{\text {alg }}$ split iff $\left(G_{K}^{\prime}\right)^{0}$ is commutative.

Proof. Let $L_{1}$ be the compositum $K^{a l g} L$. Then $L_{1}$ is the Picard-Vessiot extension of $K^{a l g}$ for the equation $(*)$, with Katz group $\left(G_{K}^{\prime}\right)^{0}$. Now apply the previous corollary.

These results give a cheap way of producing split but non- $K$-split $D$-group structures on noncommutative connected algebraic groups over $C$ (for suitable $G$ and algebraically closed $K)$.

COROLlary 3.8. Let $G$ be a connected noncommutative algebraic subgroup of $G L(n, \mathcal{U})$, defined over some field $k$ of constants. Let $A$ be a generic (in the sense of differential fields) point over $k$ of the Lie algebra $L(G)<M_{n}(\mathcal{U})$ of $G$. Let $K$ be the algebraic closure of the differential field generated over $k$ by the coordinates of $A$, and let $s(-)=[A,-]$. Then $(G, s)$ is defined over $K$, and is absolutely split but not $K$-split.

Proof. We may assume that $A=(\partial g) g^{-1}$ for $g$ a generic point over $k$ of $G$ (in the sense of differential fields). Then $L=K(g)$ is a Picard-Vessiot extension of $K$ for the equation $\partial Y=A Y$ with Katz group $G$.

So we have established one way of obtaining split but non- $K$-split $\partial_{0}$-groups from Picard-Vessiot extensions of $K$.

Finally we will give another relationship between these two classes of objects. Our notation $(K, k, \partial Y=A Y, U, L=K(U))$ is as before. Let us first note that the solution space $V^{\partial}$ of $\partial Y=A Y$ is (the set of $\mathcal{U}$-points of) $\left(G_{a}^{n}, s_{A}\right)$ (see Example 2.6). Moreover 
the fundamental matrix of solutions $U$ is a "canonical parameter" for a (differential algebraic) isomorphism of $\left(G_{a}^{n}, s_{A}\right)$ with $\left(G_{a}^{n}, s_{0}\right)$ (multiplication by $U$ ). (To say that $U$ is a canonical parameter means that moving $U$ moves the isomorphism). It easily follows as in above arguments that $\left(G_{a}^{n}, s_{A}\right)$ is $K$-split iff $U$ has its coordinates in $K$. More generally we have:

Proposition 3.9. Let $K$ be a differential subfield of $\mathcal{U}$ with algebraically closed field $k$ of constants. Let $(G, s)$ be an absolutely split (not necessarily linear) $\partial_{0}$-group defined over $K$. Let $u \in \hat{K}$ be a canonical parameter (over $K$ ) for some differential algebraic isomorphism between $G_{1}$ and some $\left(H, s_{0}\right)$ where $H$ is an algebraic group over $k$ and $s_{0}$ is the trivial connection. Then

(i) $L=K<u>$ (the differential field generated over $K$ by $u$ ) is a Picard-Vessiot extension of $K$ whose Katz group is an algebraic subgroup of $A u t(G)$.

(ii) $(G, s)$ is $K$-split iff $L=K$.

(iii) The "map" taking $(G, s)$ to L establishes a functor from the class of absolutely split $\partial_{0}$-groups over $K$ (up to K-isomorphism) to the class of Picard-Vessiot extensions of $K$ (inside $\hat{K}$ or equivalently up to isomorphism over $K$ ).

(iv) The functor in (iii) is surjective.

Proof. (i) Let $G_{1}=(G, s)(\mathcal{U})$. As $G_{1}$ is absolutely split there is an isomorphism $f$ defined over $\hat{K}$ between $G_{2}=\left(H, s_{0}\right)(\mathcal{U})$ (for some algebraic group $H$ defined over $k$ ) and $G_{1}$. By elimination of imaginaries in differentially closed fields, there is some tuple $u$ from $\hat{K}$ such that $f=f_{u}$ is defined over $K<u>$ and such that for any $u^{\prime}$ realising $\operatorname{tp}(u / K), f_{u^{\prime}}=f_{u}$ iff $u=u^{\prime}$. (This is what we mean by $u$ being a canonical parameter over $K$ for $f$.) In any case we may identify $u$ with $f_{u}$, and similarly for any realisation $u_{1}$ of $r(x)=t p(u / K)$. For each such $u_{1}, u^{-1} \cdot u_{1}$ is a (definable) automorphism $g$ of $G_{2}$. $u_{1}=u . g$, so clearly $u_{1} \in K<u, C>$. As $L=K<u>$ has the same constants as $K$, it follows that $L$ is a strongly normal extension of $K$ in the sense of Kolchin [6]. To see that $L$ is a Picard-Vessiot extension of $K$ it is enough to show that the (extrinsic) Galois group of $L$ over $K$ is linear. Working in $\mathcal{U}$, this extrinsic Galois group $G_{3}$ say, is the set of $C$-points of an algebraic group defined over $k$. Clearly $G_{3}$ acts definably (over $k$ ) and faithfully on $G_{2}$ as (group) automorphisms. As all this is going on inside the constants $C$, the action is rational. Thus the connected component of $G_{3}$ embeds (rationally) over $k$ into (the group of $C$-points of) $G L(L)$ where $L$ is the Lie algebra of $G_{2}$. Thus the connected component of $G_{3}$, and so $G_{3}$ itself, is definably the group of $C$-points of a linear algebraic group over $k$. So $L$ is a Picard-Vessiot extension of $K$. The element $u$ gives an isomorphism between $G_{3}$ and a $K$-definable subgroup $G_{4}$ of $A u t\left(G_{1}\right)$. $G_{4}$ (or rather its group of $\hat{K}$-points) is the intrinsic Galois group of $L$ over $K$. It is not hard to see that the Katz group is a $K$-algebraic subgroup $G^{\prime}$ of $A u t(G)$. (That is, $G_{4}$ is the $\partial_{0}$-group $\left(G_{1}^{\prime}, s\right)$ for a suitable connection $s$.)

(ii) Note that $L$ depends only on $G_{1}$ (not on $G_{2}$ or the isomorphism $f_{u}$ ): if $g_{w}$ were an isomorphism of $G_{1}$ with another $\partial_{0}$ group $G_{2}^{\prime}$ of the form $\left(H^{\prime}, s_{0}\right)\left(H^{\prime}\right.$ defined over $\left.k\right)$, where $w \in \hat{K}$ is a canonical parameter for $g_{w}$, then the induced isomorphism between $G_{2}$ and $G_{2}^{\prime}$ "lives in" $k$, whence $K<w>=K<u>=L$. So $G_{1}$ is $K$-split iff $u \in K$ iff $L=K$. 
(iii) If $G_{1}^{\prime}$ is another absolutely split $\partial_{0}$-group over $K$ which is isomorphic over $K$ to $G_{1}$, and $w \in \hat{K}$ is a canonical parameter for an isomorphism witnessing the splitting, then as in (ii) but using also the isomorphism between $G_{1}$ and $G_{1}^{\prime}$, we see that $K(w)=K(u)$. So we get a map $F$ from $K$-isomorphism types of absolutely split $\partial_{0}$-groups over $K$ to Picard-Vessiot extensions of $K$. To say that this is a functor means that if $f$ is an embedding of $G_{1}$ into $G_{2}$ defined over $K$ then the P-V extension of $K$ corresponding to $G_{1}$ is a subfield of that corresponding to $G_{1}^{\prime}$. This is clear from the construction of $L$ and above remarks.

(iv) Finally by the remarks preceding the proposition, any Picard-Vessiot extension of $K$ is in the image of $F$.

COROllary 3.10. Let $(K, \partial)$ be a differential field with algebraically closed field of constants. Then the following are equivalent:

(i) K has no proper Picard-Vessiot extensions.

(ii) Any $\partial_{0}$-group defined over $K$ which is absolutely split is already $K$-split.

I will end with some remarks about general $\partial_{0}$-groups (not necessarily absolutely split). Let $K$ be an algebraically closed differential field. Let $G$ be a $\partial_{0}$-group defined over $K$. Call $G K$-good if $G(K)=G(\hat{K})$. A $K$-form of $G$ is a $\partial_{0}$-group over $K$ which is isomorphic (but not necessarily over $K$ ) to $G$. We then have the following strengthening of Corollary 3.10:

Proposition 3.11. The following are equivalent:

(i) K has no proper Picard-Vessiot extensions.

(ii) Any $K$-good $\partial_{0}$-group has a unique $K$-form up to $K$-isomorphism.

Explanation. We work with definability in the differentially closed field $\mathcal{U}$. Let $G$ be a $K$-good connected $\partial_{0}$-group (so also $K$-definable). Let $G_{1}$ be a $K$-form of $G$. So $G_{1}$ is a $\partial_{0}$-group over $K$ which is definably isomorphic to $G$. Let $u \in \hat{K}$ be a canonical parameter for a definable isomorphism (which we also call $u$ ) between $G$ and $G_{1}$. All we have to do is show that $L=K\langle u\rangle$ is a Picard-Vessiot extension of $K$. First note that as $K$ is algebraically closed and $K<L<\hat{K}$,

(a) $K<u>$ has the same constants as $K$.

Next we want to show that $L$ is a differential Galois extension of $K$ in the sense of [9]. By that paper it suffices to see that the set $X$ of realizations of $t p(u / K)$ is a principal homogeneous space for a $\partial_{0}$-group $H$ defined over $K$ such that $H(\hat{K})=H(K)$. Well, for any $w$ realizing $t p(u / K)$, clearly $w=u \circ f$ for a unique definable automorphism $f$ of $G$. Let $H$ be the (definable) group of automorphisms of $G$ obtained this way. $H$ can be considered as a $\partial_{0}$-group defined over $K$, and as $G(\hat{K})=G(K)$, also $H(\hat{K})=H(K)$. So we see

(b) $L$ is a differential Galois extension of $K$ (in the sense of [9]) and moreover $\operatorname{Aut}(L / K)$ is isomorphic to $H(\hat{K})$.

Finally (by [9]) we need to see that the $\partial_{0}$-group $H$ is definably isomorphic to the group of $\mathcal{C}$-points of a linear algebraic group defined over $\mathcal{C}$. We use the differential Lie 
algebra $L^{\partial}(G)$ as introduced by Kolchin in Chapter VIII of [7]. As $G$ is a $\partial_{0}$-group, $L^{\partial}(G)$ is a finite-dimensional vector space over $\mathcal{C}$. The $\partial_{0}$-group $H$ (a group of definable automorphisms of $G$ ) embeds in $G L\left(L^{\partial}(G)\right)$ giving us what we want. (We have just observed here that if $G$ is any connected $\partial_{0}$-group and $H$ is a $\partial_{0}$-group which acts definably on $G$ as a group of automorphisms, then $H$ definably embeds in $G L_{n}(\mathcal{C})$ for some $n$.) Thus $L$ is a Picard-Vessiot extension of $K$, and is a proper extension of $K$ just if $G_{1}$ is not definably isomorphic over $K$ to $G$.

\section{References}

[1] D. Bertrand, Review of "Lectures on differential Galois theory" by A. Magid, Bull. AMS 33 (1996), 289-94.

[2] A. Buium, Differential Algebraic Groups of Finite Dimension, Lecture Notes in Math. 1509, Springer-Verlag, 1992.

[3] P. Deligne, Catégories tannakiennes, in: Grothendieck Festschrift, vol. II, P. Cartier et al. (eds.), Birkhäuser, 1990, 111-195.

[4] E. Hrushovski, Contributions to stable model theory, Ph.D. thesis, Berkeley, 1986.

[5] N. Katz, A conjecture in the arithmetic theory of differential equations, Bull. Soc. Math. France 110 (1982), 203-239.

[6] E. Kolchin, Differential Algebra and Algebraic Groups, Academic Press, 1973.

[7] E. Kolchin, Differential Algebraic Groups, Academic Press, 1985.

[8] D. Marker, Manin kernels, in: Connections between Model Theory and Algebraic and Analytic Geometry, A. Macintyre (ed.), Quaderni di matematica, vol. 6, Università di Napoli, 2000.

[9] A. Pillay, Differential Galois theory I, Illinois J. Math. 42 (1998), 678-699.

[10] A. Pillay, Geometric Stability Theory, Oxford University Press, 1996.

[11] A. Pillay, Some foundational questions concerning differential algebraic groups, Pacific J. Math. 179 (1997), 181-192.

[12] B. Poizat, Une théorie de Galois imaginaire, J. Symbolic Logic 48 (1983), 1151-1170. 